\title{
Association Between Different Versions of the Model for End-Stage Liver Disease Score and Contrast-Associated Acute Kidney Injury in Patients Undergoing Elective Percutaneous Coronary Intervention
}

\author{
Hao-ming He, MD; Chen He, MD; Zhe-bin You, MD; Si-cheng Zhang, MD; \\ Xue-qin Lin, MD; Man-qing Luo, MD; Mao-qing Lin, MD; Li-wei Zhang, MD; \\ Yan-song Guo, MD, PhD; Kai-yang Lin, MD, PhD
}

\begin{abstract}
Background: Pre-procedure liver dysfunction was associated with acute kidney injury after percutaneous coronary intervention $(\mathrm{PCl})$. The aim of this study is to assess and compare the predictive value of different liver function scoring systems for contrastassociated acute kidney injury (CA-AKI) in patients undergoing elective $\mathrm{PCI}$.
\end{abstract}

Methods and Results: A total of 5,569 patients were retrospectively enrolled. The model for end-stage liver disease (MELD) including albumin (MELD-Albumin) score (AUC=0.661) had the strongest predictive value in comparison to the MELD score (AUC=0.627), the MELD excluding the international normalized ratio (MELD-XI) score (AUC=0.560), and the MELD including sodium (MELD-Na) score $(A \cup C=0.652)$. In the fully adjusted logistic regression model, the MELD-Albumin score and the MELD-Na score were independently associated with CA-AKI regardless of whether they were treated as continuous or categorical variables; however, this was not the case for the MELD score and the MELD-XI score. Furthermore, the addition of the MELD-Albumin score significantly improved the reclassification beyond the fully adjusted logistic regression model. The study further explored the association between different versions of the MELD score and CA-AKI using restricted cubic splines and found a linear relationship between the MELD-Albumin score and the risk of CA-AKI.

Conclusions: The MELD-Albumin score had the highest predictive value for CA-AKI in patients undergoing elective PCI. The addition of the MELD-Albumin score to the existing risk prediction model significantly improved the reclassification for CA-AKI.

Key Words: Albumin; Contrast-associated acute kidney injury; Model for end-stage liver disease; Percutaneous coronary intervention

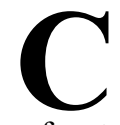
ontrast-associated acute kidney injury (CA-AKI) is one of the most common complications after percutaneous coronary intervention (PCI), which refers to acute renal impairment caused by the endovascular application of contrast medium. It is associated with prolonged hospitalization, higher medical costs, persistent kidney impairment, dialysis, and increased mortality. ${ }^{1-3}$
Therefore, accurate identification of patients at high risk of developing CA-AKI before the procedure is of great significance for improving patient prognosis.

Patients with coronary artery disease complicated with liver dysfunction are often accompanied by an increased prevalence of acute kidney injury (AKI) after PCI. ${ }^{4,5}$ Thus, assessing the liver function and determining risk stratification

Received September 21, 2021; revised manuscript received October 23, 2021; accepted November 10, 2021; J-STAGE Advance Publication released online December 21, 2021 Time for primary review: 17 days

Department of Cardiology, Shengli Clinical Medical College of Fujian Medical University, Fujian Provincial Hospital, Fuzhou (H.-m.H., C.H., S.-c.Z., X.-q.L., M.-q. Luo, M.-q. Lin, L.-w.Z., Y.-s.G., K.-y.L.); Fujian Provincial Key Laboratory of Cardiovascular Disease, Fujian Cardiovascular Institute, Fujian Provincial Center for Geriatrics, Fujian Clinical Medical Research Center for Cardiovascular Diseases, Fuzhou (H.-m.H., C.H., S.-c.Z., X.-q.L., M.-q. Luo, M.-q. Lin, L.-w.Z., Y.-s.G., K.-y.L.); Fujian Heart Failure Center Alliance, Fuzhou (H.-m.H., C.H., S.-c.Z., X.-q.L., M.-q. Luo, M.-q. Lin, L.-w.Z., Y.-s.G., K.-y.L.); and Department of Geriatric Medicine, Shengli Clinical Medical College of Fujian Medical University, Fujian Provincial Hospital, Fujian Provincial Center for Geriatrics, Fuzhou (Z.-b.Y.), China

The first three authors are considered co-first authors (H.-m.H., C.H., Z.-b.Y.).

Mailing address: Kai-yang Lin, MD, PhD and Yan-song Guo, MD, PhD, Department of Cardiology, Shengli Clinical Medical College of Fujian Medical University, Fujian Provincial Hospital, Fujian Provincial Key Laboratory of Cardiovascular Disease, Fuzhou 350001, China. E-mail: 1ky7411@sina.com (K.-y.L.); ysguo1234@126.com (Y.-s.G.)

All rights are reserved to the Japanese Circulation Society. For permissions, please e-mail: cj@j-circ.or.jp

ISSN-1346-9843 
before the procedure is a critical step in optimizing patient management. The model for end-stage liver disease (MELD) score, which is a recognized liver function assessment tool, has been proven to be associated with AKI.6,7 Furthermore, numerous studies focused on the modified MELD versions and have demonstrated that the MELD excluding the international normalized ratio (MELD-XI) score and the MELD including sodium (MELD-Na) score was also correlated with AKI. ${ }^{8-10}$ However, the predictive value of the MELD including albumin (MELD-Albumin) score for CA-AKI is still unclear. Moreover, there is a lack of research comparing the predictive ability of the MELD score and its modifications for CA-AKI. Consequently, the purpose of the present study was to investigate and compare the predictive value of the MELD score and its modifications for predicting $\mathrm{CA}-\mathrm{AKI}$ in patients undergoing elective PCI.

\section{Methods}

\section{Study Population}

The present study was designed as an observational, retrospective study. All patients who underwent elective PCI between January 2012 and December 2018 at Fujian Provincial Hospital were consecutively included in the study and retrospectively analyzed. The exclusion criteria were listed as follows: (1) patients with end-stage renal disease (estimated glomerular filtration rate $[\mathrm{eGFR}]<15 \mathrm{~mL} / \mathrm{min} / 1.73 \mathrm{~m}^{2}$ ) or those who required long-term dialysis; (2) patients who had been administrated with contrast agents within 1 week before or $48 \mathrm{~h}$ after the procedure; (3) patients who took nephrotoxic drugs within 1 week before or $48 \mathrm{~h}$ after the procedure; (4) patients who had missing data on total bilirubin, international normalized ratio (INR), serum sodium, albumin, and pre- or post-procedural serum creatinine (SCr); and (5) patients who died within the first $24 \mathrm{~h}$ after hospital admission. Finally, 5,569 participants were included in the analysis. This study was conducted in accordance with the Declaration of Helsinki and ethical approval was obtained from the Fujian Provincial Hospital ethics committee (Ethical approval number: K2019-07-011). All patients provided written informed consent.

\section{Protocol}

All demographic, clinical, laboratory, and angiographic data were collected via electronic medical records. Venous blood samples were collected on admission or the second day after admission. The SCr concentration was measured before the procedure and consecutive 2 days after the procedure. All patients received non-ionic, low-osmolality contrast medium (either Iopamiron or Ultravist, both $370 \mathrm{mgI} / \mathrm{mL}$ ) during PCI procedures, which were conducted by experienced interventional cardiologists. An intravenous infusion of $0.9 \%$ saline at a rate of $1 \mathrm{~mL} / \mathrm{kg} / \mathrm{h}$ for $12 \mathrm{~h}$ was performed for all patients throughout the perioperative period (or $0.5 \mathrm{~mL} / \mathrm{kg} / \mathrm{h}$ among heart failure patients). The choice of pharmaceutical therapy was at the discretion of the treating physician, in accordance with appropriate guidelines.

\section{Definitions and Study Endpoint}

The study endpoint was the development of CA-AKI, which was defined as an increase in $\mathrm{SCr} \geq 0.3 \mathrm{mg} / \mathrm{dL}$ or $\geq 50 \%$ within $48 \mathrm{~h}$ after the administration of contrast media. ${ }^{11}$ Anemia was defined as a hematocrit $<0.39$ (male) or $<0.36$ (female). We calculated the standard MELD score and its
3 modifications (MELD-XI score, MELD-Na score, and MELD-Albumin score) according to the following formulas: (1) $\mathrm{MELD}=3.78 \times \ln ($ total bilirubin, $\mathrm{mg} / \mathrm{dL})+11.2 \times$ $\ln (\mathrm{INR})+9.57 \times \ln (\mathrm{SCr}, \mathrm{mg} / \mathrm{dL})+6.43$; (2) MELD-XI $=$ $5.11 \times \ln ($ total bilirubin, $\mathrm{mg} / \mathrm{dL})+11.76 \times \ln (\mathrm{SCr}, \mathrm{mg} / \mathrm{dL})+$ 9.44; (3) MELD-Na = MELD - (serum sodium, mmol/L) $-0.025 \times$ MELD $\times(140-$ serum sodium, $\mathrm{mmol} / \mathrm{L})+140 ;(4)$ MELD-Albumin $=3.78 \times \ln ($ total bilirubin, $\mathrm{mg} / \mathrm{dL})+11.2 \times$ $\ln [1+(4.1-$ Albumin, $\mathrm{g} / \mathrm{dL})]+9.57 \times \ln (\mathrm{SCr}, \mathrm{mg} / \mathrm{dL})+6.43$ (if the value of albumin was $<4.1 \mathrm{~g} / \mathrm{dL}$ ) or MELDAlbumin $=3.78 \times \ln ($ total bilirubin, $\mathrm{mg} / \mathrm{dL})+11.2 \times \ln (1)+$ $9.57 \times \ln (\mathrm{SCr}, \mathrm{mg} / \mathrm{dL})+6.43$ (if the value of albumin was $>4.1 \mathrm{~g} / \mathrm{dL}$ ). ${ }^{12-14}$ To avoid a negative score, the lower limit of all parameters was set at 1.0 . The serum sodium values $\leq 125 \mathrm{mmol} / \mathrm{L}$ will be set to $125 \mathrm{mmol} / \mathrm{L}$, and values $\geq 140 \mathrm{mmol} / \mathrm{L}$ will be set to $140 \mathrm{mmol} / \mathrm{L}$. The eGFR was calculated using the modified Modification of Diet in Renal Disease equation: eGFR $=186.3 \times(\mathrm{SCr})^{-1.154} \times(\mathrm{age})^{-0.203} \times$ 0.742 (if the patient was female). ${ }^{15}$

\section{Statistical Analysis}

Statistical analyses were conducted using the R statistical language (R Foundation for Statistical Computing, Vienna, Austria; version 4.0.2). Continuous variables were presented in the form of mean \pm standard deviation (SD) or median (interquartile range) and analyzed using the Student's t-test (normally distributed) or Mann-Whitney U-test (non-normally distributed). Categorical variables were summarized as counts (percentage) and compared using the chi-squared test or Fisher's exact test.

The association between different versions of the MELD score and CA-AKI was evaluated by logistic analysis using 3 multivariate models. The significative variables determined by univariate logistic analysis based on a significance threshold of $\mathrm{P}$ value $<0.05$ and those of clinical importance were incorporated into multivariate analysis. Model 1 was adjusted for age $>75$ years and gender. Model 2 included variables from Model 1 plus diabetes, hypertension, history of myocardial infarction (MI), anemia, left ventricular ejection fraction $(\mathrm{LVEF})<40 \%$, chronic kidney disease (CKD), hyperuricemia, acute MI, contrast volume $>150 \mathrm{~mL}$, and multivessel disease. Model 3 was further adjusted for variables used in Model 2 and added in white blood cell (WBC), high-density lipoprotein-cholesterol (HDL-C), and glucose. For the subgroup analyses, the presence of interaction was determined across various subgroups. To further explore the potential linear and non-linear associations between different versions of the MELD score and the odds ratio (OR) of CA-AKI after adjusting for confounding factors, as Model 3 used in the logistic analysis, restricted cubic splines with 3 knots were used.

According to the receiver operating characteristic curve (ROC) analysis, the optimal cut-off values of the MELD score and its modifications for predicting CA-AKI were determined by maximizing the Youden index. The area under the curve (AUC), continuous net reclassification improvement (NRI), and integrated discrimination improvement (IDI) values were used to compare the predictive power of the MELD score and its modifications on CA-AKI. Differences in AUCs were compared using the DeLong method. In addition, we calculated the $\mathrm{C}$-statistics, continuous NRI, and IDI to examine the incremental effects of the MELD score and its 3 modifications on the predictive potential of Model 3. Two-sided $\mathrm{P}<0.05$ was considered statistically significant. 
Table 1. Baseline Characteristics of the Study Population

\begin{tabular}{|c|c|c|c|c|}
\hline Variables & $\begin{array}{c}\text { Total } \\
(n=5,569)\end{array}$ & $\begin{array}{c}\text { Non-CA-AKI } \\
(n=5,218)\end{array}$ & $\begin{array}{l}\text { CA-AKI } \\
(n=351)\end{array}$ & $P$ value \\
\hline \multicolumn{5}{|l|}{ Demographics } \\
\hline Age, years & $65.4 \pm 10.5$ & $65.1 \pm 10.4$ & $68.6 \pm 11.2$ & $<0.001$ \\
\hline Age $>75$ years, $n(\%)$ & $989(17.8)$ & $884(16.9)$ & 105 (29.9) & $<0.001$ \\
\hline Sex, female, n (\%) & $1,194(21.4)$ & $1,089(20.9)$ & 105 (29.9) & $<0.001$ \\
\hline $\mathrm{BMI}, \mathrm{kg} / \mathrm{m}^{2}$ & $24.45 \pm 3.24$ & $24.47 \pm 3.24$ & $24.12 \pm 3.34$ & 0.077 \\
\hline \multicolumn{5}{|l|}{ Medical history, n (\%) } \\
\hline History of MI & $734(13.2)$ & $692(13.3)$ & $42(12.0)$ & 0.540 \\
\hline Diabetes & $1,977(35.5)$ & $1,823(34.9)$ & $154(43.9)$ & 0.001 \\
\hline Hypertension & $3,782(67.9)$ & $3,515(67.4)$ & $267(76.1)$ & 0.001 \\
\hline Acute MI & $1,757(31.5)$ & $1,552(29.7)$ & 205 (58.4) & $<0.001$ \\
\hline Anemia & $1,525(27.4)$ & $1,370(26.3)$ & $155(44.2)$ & $<0.001$ \\
\hline CKD & $548(9.8)$ & $479(9.2)$ & $69(19.7)$ & $<0.001$ \\
\hline Hyperuricemia & $1,709(30.7)$ & $1,578(30.2)$ & $131(37.3)$ & 0.006 \\
\hline \multicolumn{5}{|l|}{ Procedure performed } \\
\hline Multivessel disease, n (\%) & $4,473(83.3)$ & $4,178(83.0)$ & $295(87.8)$ & 0.027 \\
\hline Number of stents & $1.64 \pm 0.88$ & $1.64 \pm 0.87$ & $1.67 \pm 0.97$ & 0.595 \\
\hline Stent length, mm & $44.62 \pm 26.92$ & $44.53 \pm 26.80$ & $45.88 \pm 28.62$ & 0.372 \\
\hline Contrast volume >150 mL, n (\%) & $3,860(72.4)$ & 3,617 (72.3) & $243(73.6)$ & 0.647 \\
\hline \multicolumn{5}{|c|}{ Medical therapy during hospitalization, n (\%) } \\
\hline Antiplatelet & $5,556(99.8)$ & $5,207(99.8)$ & $349(99.4)$ & 0.437 \\
\hline Statin & $5,519(99.1)$ & $5,172(99.1)$ & 347 (98.9) & 0.839 \\
\hline ACEI/ARB & $4,602(82.6)$ & $4,300(82.4)$ & $302(86.0)$ & 0.096 \\
\hline$\beta$-blocker & $4,602(82.6)$ & $4,299(82.4)$ & $303(86.3)$ & 0.070 \\
\hline $\mathrm{CCB}$ & $1,965(35.3)$ & $1,830(35.1)$ & $135(38.5)$ & 0.219 \\
\hline Warfarin & $79(1.5)$ & $68(1.4)$ & $11(3.5)$ & 0.006 \\
\hline \multicolumn{5}{|l|}{ Laboratory measurements } \\
\hline Serum creatinine, mg/dL & $0.92 \pm 0.28$ & $0.92 \pm 0.27$ & $0.94 \pm 0.38$ & 0.136 \\
\hline eGFR, mL/min/1.73 m² & $89.64 \pm 24.21$ & $89.55 \pm 23.17$ & $90.98 \pm 36.36$ & 0.285 \\
\hline WBC, $109 / \mathrm{L}$ & $7.38 \pm 2.33$ & $7.33 \pm 2.28$ & $7.99 \pm 2.87$ & $<0.001$ \\
\hline Platelet, 109/L & $222.56 \pm 68.71$ & $222.72 \pm 69.04$ & $220.28 \pm 63.57$ & 0.521 \\
\hline Albumin, g/dL & $4.13 \pm 0.41$ & $4.15 \pm 0.40$ & $3.89 \pm 0.46$ & $<0.001$ \\
\hline Total bilirubin, mmol/L & $0.60(0.44-0.82)$ & $0.60(0.44-0.81)$ & $0.58(0.45-0.88)$ & 0.526 \\
\hline $\mathrm{ALT}, \mathrm{U} / \mathrm{L}$ & $23.00(16.00-35.00)$ & $23.00(16.00-35.00)$ & $23.00(15.00-37.00)$ & 0.865 \\
\hline AST, U/L & $22.00(17.00-31.00)$ & $21.00(17.00-30.00)$ & $25.00(18.00-51.00)$ & $<0.001$ \\
\hline ALP, U/L & $72.00(60.00-88.00)$ & $72.00(60.00-88.00)$ & $73.00(61.75-88.50)$ & 0.304 \\
\hline GGT, U/L & $32.00(21.00-50.00)$ & $32.00(21.00-50.00)$ & $34.00(21.00-54.25)$ & 0.449 \\
\hline Cholesterol, mg/dL & $163.57 \pm 44.86$ & $163.19 \pm 44.86$ & $165.12 \pm 43.70$ & 0.459 \\
\hline Triglyceride, mmol/L & 122.19 (91.20-172.66) & 122.19 (91.20-173.55) & $122.19(90.32-165.58)$ & 0.676 \\
\hline HDL-C, mg/dL & $40.22 \pm 11.21$ & $40.22 \pm 11.21$ & $38.67 \pm 10.05$ & 0.019 \\
\hline LDL-C, mg/dL & $105.18 \pm 39.06$ & $104.80 \pm 39.06$ & $107.12 \pm 38.67$ & 0.319 \\
\hline Glucose, mg/dL & $110.16 \pm 36.18$ & $109.44 \pm 35.46$ & $118.98 \pm 45.90$ & $<0.001$ \\
\hline INR & $1.03 \pm 0.17$ & $1.02 \pm 0.17$ & $1.06 \pm 0.20$ & $<0.001$ \\
\hline Serum sodium, mmol/L & $140.15 \pm 3.58$ & $140.23 \pm 3.51$ & $138.85 \pm 4.34$ & $<0.001$ \\
\hline MELD score & $6.88(6.43-7.90)$ & $6.87(6.43-7.83)$ & $7.60(6.65-8.96)$ & $<0.001$ \\
\hline MELD-XI score & $9.44(9.44-10.28)$ & $9.44(9.44-10.21)$ & $9.44(9.44-11.47)$ & $<0.001$ \\
\hline MELD-Na score & $7.61(6.60-9.58)$ & $7.57(6.54-9.40)$ & 9.09 (7.37-11.82) & $<0.001$ \\
\hline MELD-Albumin score & $7.39(6.43-9.59)$ & $7.28(6.43-9.37)$ & $9.11(7.02-12.17)$ & $<0.001$ \\
\hline LVEF $<40 \%$, n (\%) & $155(2.9)$ & $128(2.6)$ & $27(8.0)$ & $<0.001$ \\
\hline
\end{tabular}

ACEI/ARB, angiotensin-converting enzyme inhibitor/angiotensin receptor blocker; ALP, alkaline phosphatase; ALT, alanine aminotransferase; AST, aspartate aminotransferase; BMI, body mass index; CA-AKI, contrast-associated acute kidney injury; CCB, calcium channel blockers; CKD, chronic kidney disease; eGFR, estimated glomerular filtration rate; GGT, gamma-glutamyl transpeptidase; HDL-C, high-density lipoprotein-cholesterol; INR, international normalized ratio; LDL-C, low-density lipoprotein-cholesterol; LVEF, left ventricular ejection fraction; MI, myocardial infarction; MELD, model for end-stage liver disease; MELD-Albumin, model for end-stage liver disease including albumin; MELD$\mathrm{Na}$, model for end-stage liver disease including sodium; MELD-XI, model for end-stage liver disease excluding the international normalized ratio; WBC, white blood cell. 


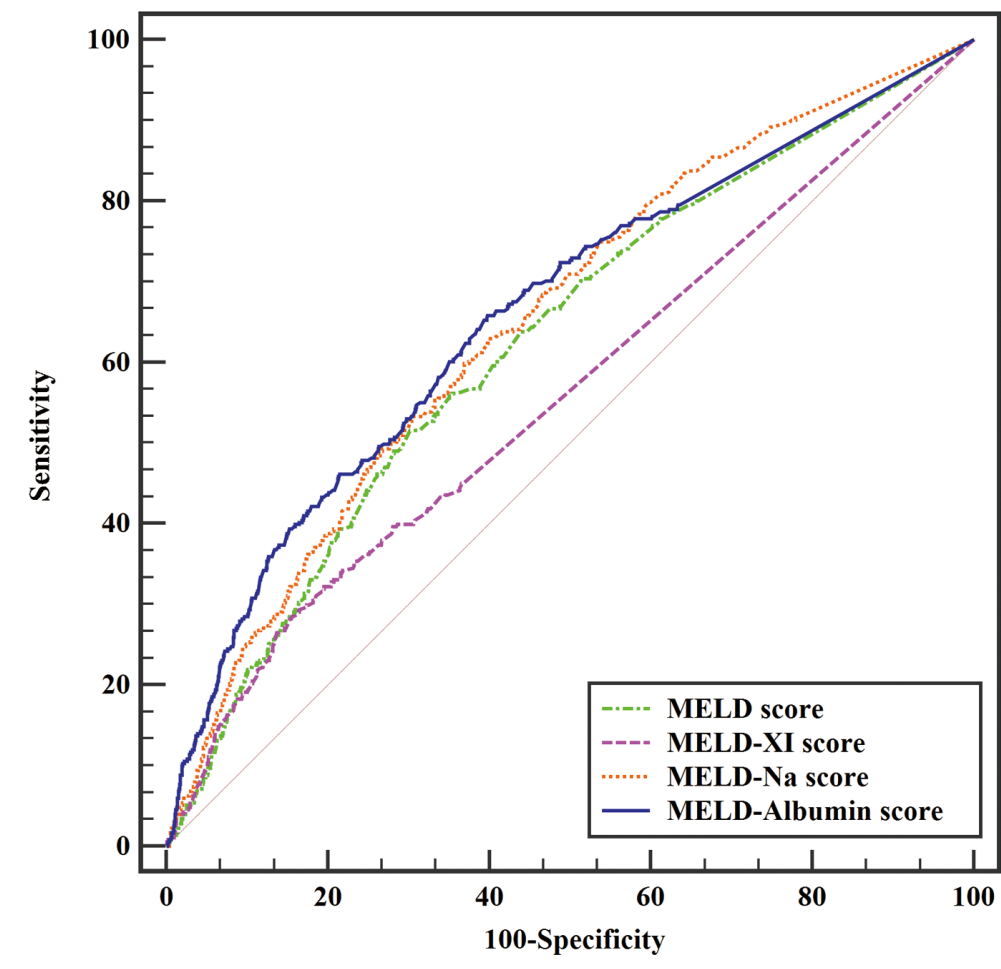

Figure 1. Receiver operating characteristic curve analysis of the MELD score and its modifications for predicting CA-AKI. CA-AKI, contrast-associated acute kidney injury; MELD, model for end-stage liver disease; MELD-Albumin, model for end-stage liver disease including albumin; MELD-Na, model for end-stage liver disease including sodium; MELD-XI, model for end-stage liver disease excluding the international normalized ratio.

\begin{tabular}{|c|c|c|c|c|c|c|}
\hline & AUC & $P$ value & $\begin{array}{c}\text { Continuous NRI } \\
(95 \% \mathrm{Cl})\end{array}$ & $P$ value & IDI (95\% CI) & $P$ value \\
\hline MELD-Albumin vs. MELD & 0.661 vs. 0.627 & 0.022 & $0.448(0.340-0.555)$ & $<0.001$ & $0.018(0.013-0.023)$ & $<0.001$ \\
\hline MELD-Albumin vs. MELD-XI & 0.661 vs. 0.560 & $<0.001$ & $0.485(0.377-0.592)$ & $<0.001$ & $0.020(0.015-0.024)$ & $<0.001$ \\
\hline MELD-Albumin vs. MELD-Na & 0.661 vs. 0.652 & 0.556 & $0.308(0.200-0.415)$ & $<0.001$ & $0.010(0.004-0.016)$ & $<0.001$ \\
\hline
\end{tabular}

AUC, area under the curve; $\mathrm{Cl}$, confidence interval; IDI, integrated discrimination improvement; NRI, net reclassification improvement. Other abbreviations as in Table 1.

\section{Results}

\section{Patients Characteristics}

Among the 5,569 patients enrolled, 351 (6.3\%) patients developed CA-AKI. The mean age of our study population was $65.4 \pm 10.5$ years and $989(17.8 \%)$ patients were aged $>75$ years. Most patients were men (78.6\%) and 734 $(13.2 \%)$ had a history of MI. Patients who developed CAAKI were more likely to have acute MI, anemia, CKD, hyperuricemia, multivessel disease, and a history of diabetes and hypertension. They also had higher levels of WBC and glucose, but lower levels of albumin, HDL-C, serum sodium, and LVEF. The MELD score, MELD-XI score, MELD-Na score, and MELD-Albumin score were higher in patients with CA-AKI than those without CA-AKI. More information on the baseline characteristics is detailed in Table 1.

\section{Predictive Value of the MELD Score and Its 3 Modifications on CA-AKI}

According to the ROC analysis, the optimal cut-off value of the MELD score, MELD-XI score, MELD-Na score, and MELD-Albumin score for predicting CA-AKI were 7.6, 11.2, 8.2, and 7.9, respectively. The MELD-Albumin score had higher AUC values than the MELD score (0.661 vs. $0.627, \mathrm{P}=0.022)$ and the MELD-XI score $(0.661$ vs. $0.560, \mathrm{P}<0.001)$, but similar AUC values to the MELD-Na score (0.661 vs. $0.652, \mathrm{P}=0.556$ ) (Figure 1). Further NRI and IDI analysis indicated that the MELD-Albumin score significantly improved the reclassification and discrimination ability for the prediction of CA-AKI beyond the MELD score, MELD-XI score, and MELD-Na score (all $\mathrm{P}<0.001$ ) (Table 2).

Effect of the MELD Score and Its 3 Modifications on CA-AKI The association between different versions of the MELD score and CA-AKI was investigated by using univariate and multivariate logistic regression models. When different versions of the MELD score were analyzed as binary variables according to their optimal cut-off values, the MELD score and its 3 modifications were independently associated with CA-AKI, regardless of which model was used for adjustment. When analyzed as continuous variables, only the MELD-Na score $(\mathrm{OR}=1.065 ; 95 \% \mathrm{CI}, 1.024-1.109$ per 


\begin{tabular}{|c|c|c|c|c|c|c|c|c|}
\hline & \multicolumn{2}{|c|}{ Unadjusted } & \multicolumn{2}{|c|}{ Model 1} & \multicolumn{2}{|c|}{ Model 2} & \multicolumn{2}{|c|}{ Model 3} \\
\hline & OR $(95 \% \mathrm{Cl})$ & $P$ value & OR $(95 \% \mathrm{Cl})$ & $P$ value & OR $(95 \% \mathrm{Cl})$ & $P$ value & OR $(95 \% \mathrm{Cl})$ & $P$ value \\
\hline \multicolumn{9}{|l|}{ MELD, continuous } \\
\hline $\begin{array}{l}\text { Per 1-point } \\
\text { increment }\end{array}$ & $\begin{array}{c}1.141 \\
(1.095-1.189)\end{array}$ & $<0.001$ & $\begin{array}{c}1.129 \\
(1.082-1.179)\end{array}$ & $<0.001$ & $\begin{array}{c}1.050 \\
(0.987-1.118)\end{array}$ & 0.125 & $\begin{array}{c}1.039 \\
(0.975-1.107)\end{array}$ & 0.234 \\
\hline \multicolumn{9}{|l|}{ MELD, categorical } \\
\hline MELD $\leq 7.6$ & Ref. & Ref. & Ref. & Ref. & Ref. & Ref. & Ref. & Ref. \\
\hline MELD > 7.6 & $\begin{array}{c}2.376 \\
(1.911-2.953)\end{array}$ & $<0.001$ & $\begin{array}{c}2.373 \\
(1.894-2.972)\end{array}$ & $<0.001$ & $\begin{array}{c}1.723 \\
(1.316-2.257)\end{array}$ & $<0.001$ & $\begin{array}{c}1.643 \\
(1.250-2.159)\end{array}$ & $<0.001$ \\
\hline \multicolumn{9}{|l|}{ MELD-XI, continuous } \\
\hline $\begin{array}{l}\text { Per 1-point } \\
\text { increment }\end{array}$ & $\begin{array}{c}1.131 \\
(1.081-1.183)\end{array}$ & $<0.001$ & $\begin{array}{c}1.124 \\
(1.073-1.177)\end{array}$ & $<0.001$ & $\begin{array}{c}1.016 \\
(0.941-1.097)\end{array}$ & 0.683 & $\begin{array}{c}1.005 \\
(0.930-1.086)\end{array}$ & 0.893 \\
\hline \multicolumn{9}{|l|}{ MELD-XI, categorical } \\
\hline MELD-XI $\leq 11.2$ & Ref. & Ref. & Ref. & Ref. & Ref. & Ref. & Ref. & Ref. \\
\hline MELD-XI >11.2 & $\begin{array}{c}2.150 \\
(1.684-2.746)\end{array}$ & $<0.001$ & $\begin{array}{c}2.155 \\
(1.673-2.777)\end{array}$ & $<0.001$ & $\begin{array}{c}1.600 \\
(1.123-2.281)\end{array}$ & 0.009 & $\begin{array}{c}1.492 \\
(1.043-2.134)\end{array}$ & 0.029 \\
\hline \multicolumn{9}{|l|}{$\begin{array}{l}\text { MELD-Na, } \\
\text { continuous }\end{array}$} \\
\hline $\begin{array}{l}\text { Per 1-point } \\
\text { increment }\end{array}$ & $\begin{array}{c}1.145 \\
(1.112-1.179)\end{array}$ & $<0.001$ & $\begin{array}{c}1.138 \\
(1.104-1.173)\end{array}$ & $<0.001$ & $\begin{array}{c}1.077 \\
(1.036-1.120)\end{array}$ & $<0.001$ & $\begin{array}{c}1.065 \\
(1.024-1.109)\end{array}$ & 0.002 \\
\hline \multicolumn{9}{|l|}{ MELD-Na, categorical } \\
\hline MELD-Na $\leq 8.2$ & Ref. & Ref. & Ref. & Ref. & Ref. & Ref. & Ref. & Ref. \\
\hline MELD-Na >8.2 & $\begin{array}{c}2.479 \\
(1.985-3.095)\end{array}$ & $<0.001$ & $\begin{array}{c}2.468 \\
(1.963-3.102)\end{array}$ & $<0.001$ & $\begin{array}{c}1.721 \\
(1.327-2.232)\end{array}$ & $<0.001$ & $\begin{array}{c}1.617 \\
(1.240-2.109)\end{array}$ & $<0.001$ \\
\hline \multicolumn{9}{|l|}{$\begin{array}{l}\text { MELD-Albumin, } \\
\text { continuous }\end{array}$} \\
\hline $\begin{array}{l}\text { Per 1-point } \\
\text { increment }\end{array}$ & $\begin{array}{c}1.193 \\
(1.157-1.231)\end{array}$ & $<0.001$ & $\begin{array}{c}1.184 \\
(1.147-1.223)\end{array}$ & $<0.001$ & $\begin{array}{c}1.105 \\
(1.057-1.156)\end{array}$ & $<0.001$ & $\begin{array}{c}1.097 \\
(1.047-1.148)\end{array}$ & $<0.001$ \\
\hline \multicolumn{9}{|l|}{$\begin{array}{l}\text { MELD-Albumin, } \\
\text { categorical }\end{array}$} \\
\hline MELD-Albumin $\leq 7.9$ & Ref. & Ref. & Ref. & Ref. & Ref. & Ref. & Ref. & Ref. \\
\hline MELD-Albumin >7.9 & $\begin{array}{c}2.900 \\
(2.310-3.640)\end{array}$ & $<0.001$ & $\begin{array}{c}2.751 \\
(2.180-3.470)\end{array}$ & $<0.001$ & $\begin{array}{c}1.712 \\
(1.300-2.254)\end{array}$ & $<0.001$ & $\begin{array}{c}1.651 \\
(1.250-2.180)\end{array}$ & $<0.001$ \\
\hline
\end{tabular}

Model 1: Adjusted for age $>75$ years and gender. Model 2: Adjusted for variables from Model 1 plus diabetes, hypertension, history of MI, anemia, LVEF $<40 \%$, CKD, hyperuricemia, acute MI, contrast volume $>150 \mathrm{~mL}$, and multivessel disease. Model 3 : Adjusted for variables from Model 2 plus WBC, HDL-C, and glucose. OR, odds ratio. Other abbreviations as in Tables 1,2.

1-point increment; $\mathrm{P}=0.002)$ and MELD-Albumin score $(\mathrm{OR}=1.097 ; 95 \%$ CI, $1.047-1.148$ per 1 -point increment; $\mathrm{P}<0.001)$ were independent risk factors for CA-AKI after adjusting for model 3 ; however, this was not the case for the MELD score $(\mathrm{OR}=1.039 ; 95 \% \mathrm{CI}, 0.975-1.107$ per 1-point increment; $\mathrm{P}=0.234)$ and the MELD-XI score $(\mathrm{OR}=1.005$; 95\% CI, 0.930-1.086 per 1-point increment; $\mathrm{P}=0.893$ ) (Table 3). In most subgroups, no significant interaction effect between different versions of the MELD score and CA-AKI was found (Figure 2 and Supplementary Figures 1-3). We further evaluated the relationship between different versions of the MELD score and the OR of CAAKI after adjusting for confounding factors that Model 3 used in logistic analysis using restricted cubic splines with $95 \%$ confidence intervals. A linear relationship between the MELD-Albumin score and the risk of CA-AKI was found (Figure 3A-D).

\section{Incremental Effects of MELD Score and Its 3 Modifications on the Predictive Potential of Model 3 for CA-AKI}

The addition of the MELD score and its 3 modifications did not have significant incremental effects on the AUC of model 3 (all $\mathrm{P}$ for comparison $>0.05$ ); however, the addition of the MELD-Albumin score significantly improved the reclassification and discrimination ability beyond model 3, with a continuous NRI of 0.438 and an IDI of 0.006 (both $\mathrm{P}<0.001$ ) (Table 4).

\section{Discussion}

In this retrospective study, we discovered that the MELDAlbumin score had the highest predictive value for predicting CA-AKI than other versions of the MELD score in patients undergoing elective PCI. There is a linear relationship between the MELD-Albumin score and the risk of CA-AKI. Furthermore, the addition of the MELD-Albumin score significantly improved the reclassification and discrimination ability beyond the fully adjusted logistic regression model.

Patients with liver dysfunction treated with PCI were more susceptible to developing AKI and this was closely related to poor prognosis. In a large, patient-level study using the national inpatient sample, an all-payer claims database in the United States, patients with cirrhosis who underwent PCI had a higher risk of AKI and in-hospital mortality than those without cirrhosis. ${ }^{4}$ Another large study also detected a higher rate of AKI, gastrointestinal bleeding, and 90-day mortality in patients with cirrhosis 


\section{MELD-Albumin score and CA-AKI}

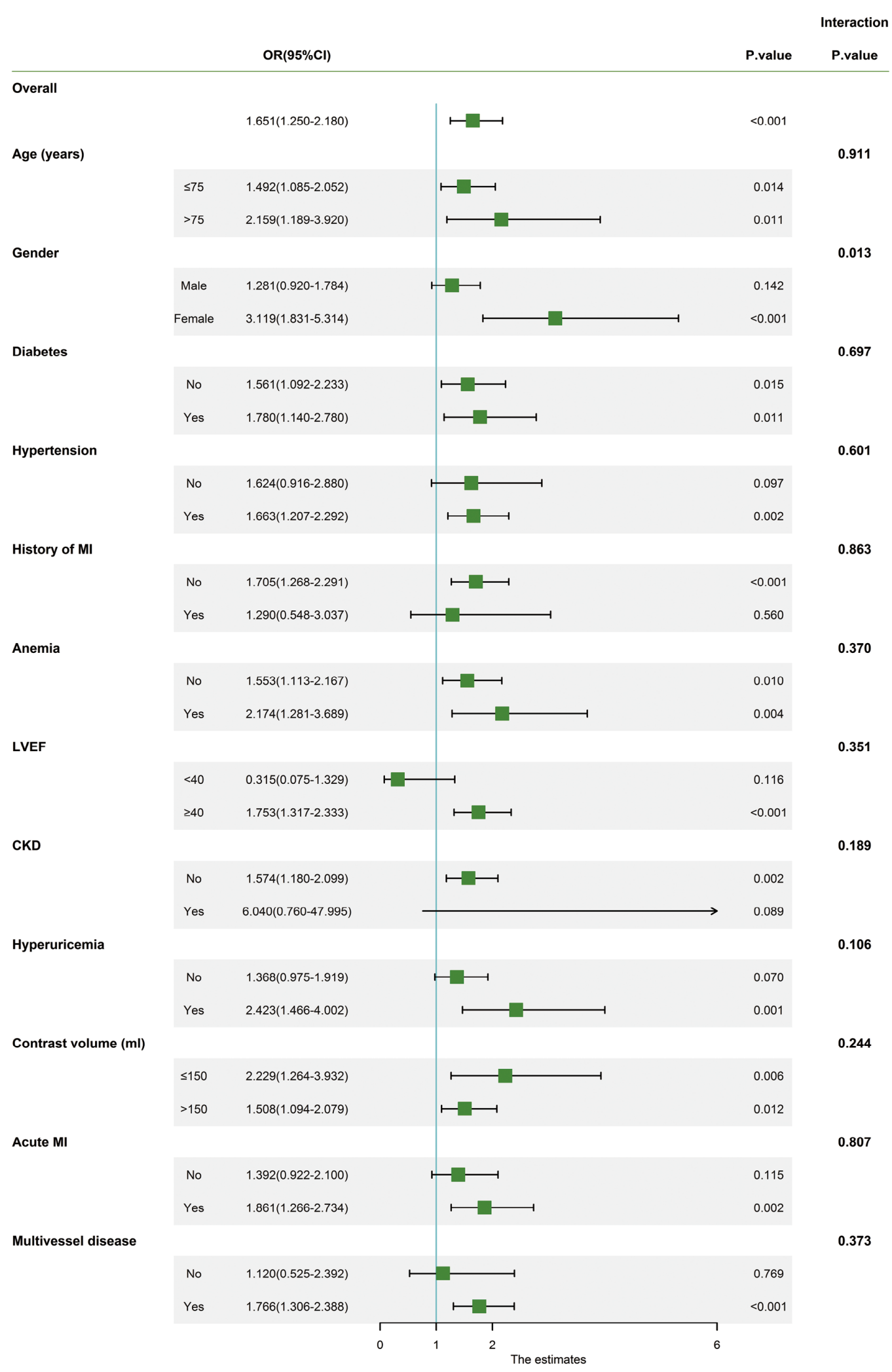

Figure 2. Forrest plots of subgroup analysis of the association between the MELD-Albumin score and CA-AKI. CA-AKI, contrastassociated acute kidney injury; MELD-Albumin, model for end-stage liver disease including albumin. 

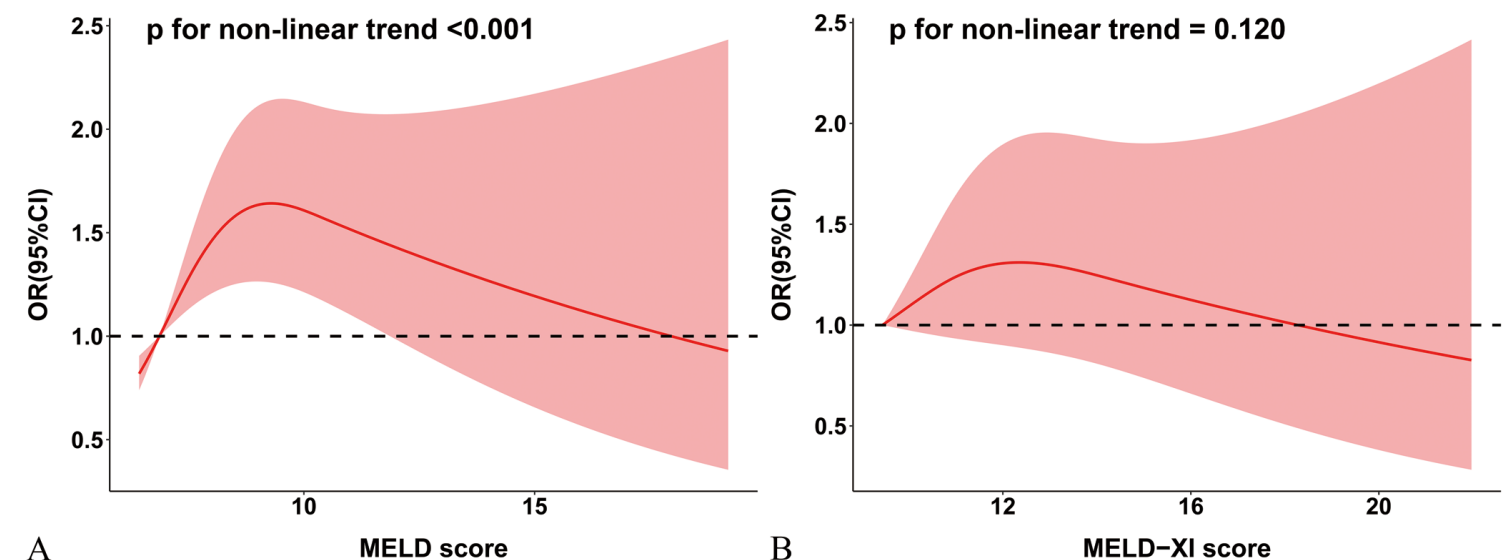

A

MELD score
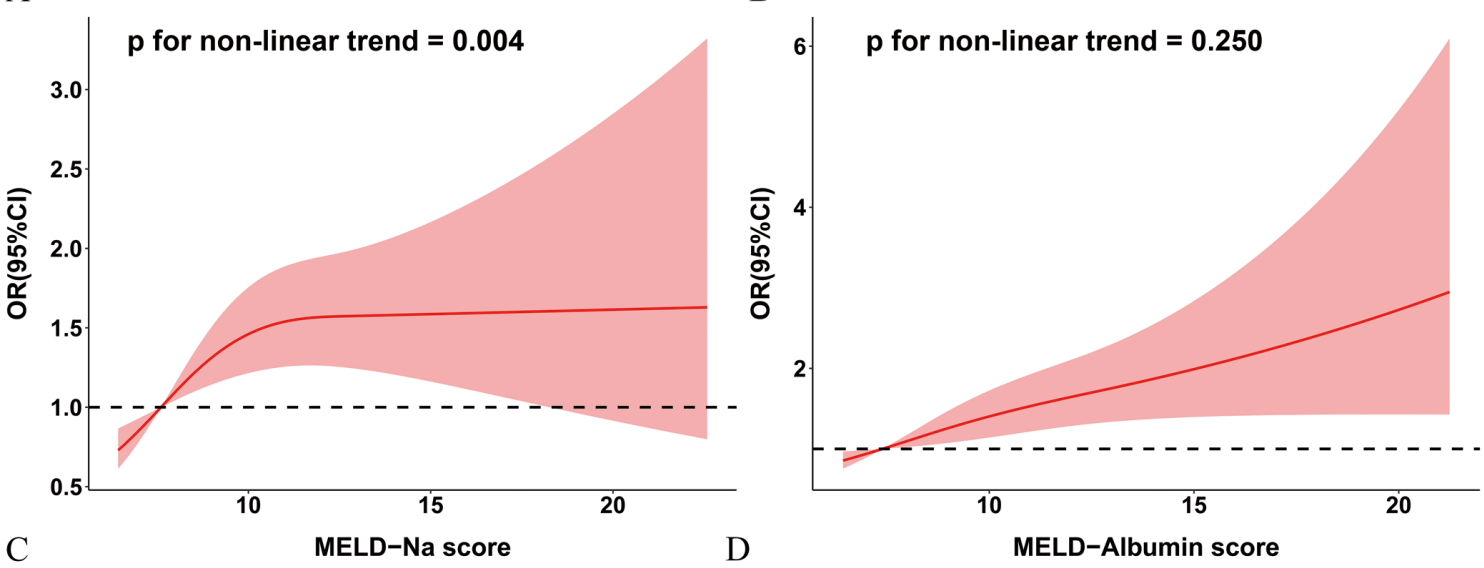

Figure 3. Restricted cubic spline curves with logistic regression between different versions of the MELD score and CA-AKI. Black dashed horizontal lines represent the odds ratio of 1.0. Red lines indicate the estimated odds ratio (OR), and the shaded ribbons represent a 95\% confidence interval $(\mathrm{Cl})$. OR (95\% Cl) were all adjusted according to Model 3 in logistic analysis. (A) Association between the MELD score and CA-AKI. (B) Association between the MELD-XI score and CA-AKI. (C) Association between the MELD-Na score and CA-AKI. (D) Association between the MELD-Albumin score and CA-AKI. CA-AKI, contrast-associated acute kidney injury; MELD, model for end-stage liver disease; MELD-Albumin, model for end-stage liver disease including albumin; MELD-Na, model for end-stage liver disease including sodium; MELD-XI, model for end-stage liver disease excluding the international normalized ratio.

\begin{tabular}{|c|c|c|c|c|c|c|}
\hline Model & C-statistic & $P$ value & $\begin{array}{c}\text { Continuous } \\
\text { NRI }\end{array}$ & $P$ value & IDI & $P$ value \\
\hline Model 3* & 0.739 & Ref. & \multicolumn{2}{|c|}{ Ref. } & \multicolumn{2}{|c|}{ Ref. } \\
\hline Model 3+MELD (categorical) & 0.745 & 0.117 & 0.314 & $<0.001$ & 0.004 & 0.069 \\
\hline Model 3+MELD-XI (categorical) & 0.741 & 0.344 & 0.276 & $<0.001$ & 0.001 & 0.396 \\
\hline Model 3+MELD-Na (categorical) & 0.745 & 0.116 & 0.354 & $<0.001$ & 0.003 & 0.108 \\
\hline Model 3+MELD-Albumin (categorical) & 0.742 & 0.414 & 0.438 & $<0.001$ & 0.006 & $<0.001$ \\
\hline
\end{tabular}

${ }^{*}$ Model 3 includes the following parameters: age $>75$ years, gender, diabetes, hypertension, history of $\mathrm{Ml}$, anemia, LVEF $<40 \%$, CKD, hyperuricemia, acute $\mathrm{MI}$, contrast volume $>150 \mathrm{~mL}$, multivessel disease, WBC, HDL-C, and glucose. Abbreviations as in Tables 1,2.

receiving PCI than those without cirrhosis. ${ }^{5}$ Consequently, assessing the liver function before the procedure has significant clinical value for optimizing patient management and improving patient prognosis.

As early as 2000, Malinchoc et al established the MELD score based on 3 commonly used clinical parameters. The MELD score was originally developed as a valuable tool for assessing liver function and had been demonstrated to be associated with poor short-term prognosis in patients with cirrhosis who received a transjugular intrahepatic portosystemic shunt. ${ }^{16}$ Recently, many studies extended the application scope of the MELD score and determined the association between the MELD score and adverse outcomes in patients who underwent PCI. ${ }^{17,18}$ Furthermore, numerous studies have focused on the predictive value of the MELD score and its modifications on AKI. A multi- 
center prospective cohort study of patients with severe alcoholic hepatitis conducted by Sujan et al discovered that a higher MELD score at admission can predict inpatient AKI. ${ }^{6}$ Tariq et al performed a meta-analysis involving 18,474 patients with cirrhosis and found that the MELD score was related to AKI. ${ }^{7}$ In addition, an increased MELD-XI score was validated to be an independent risk factor for AKI in patients with postcardiotomy cardiogenic shock treated with venoarterial extracorporeal membrane oxygenation by another retrospective study. ${ }^{8}$ Similarly, in a prospective observational study, 131 patients who underwent hepatobiliary surgeries were analyzed and it was suggested that a higher level of the MELD-Na score was significantly associated with an increased risk of developing AKI. ${ }^{9}$ Bao et al retrospectively evaluated patients who required orthotopic liver transplantation and identified a correlation between the MELD-Na score and AKI.10 However, to our knowledge, there are no studies that explored the predictive value of the MELD-Albumin score for CA-AKI. In addition, no study has directly compared the predictive value of the MELD score and its modifications for predicting CA-AKI. Our study assessed and compared the predictive value of different versions of the MELD score and showed that the MELD-Albumin score had the highest predictive value for predicting CA-AKI than the MELD score, the MELD-XI score, and the MELD-Na score in patients undergoing elective PCI. The addition, the MELD-Albumin score significantly improved the reclassification and discrimination ability beyond the fully adjusted logistic regression model, with a continuous NRI of 0.438 and an IDI of 0.006 (both $\mathrm{P}<0.001$ ). Moreover, the odds of developing CA-AKI increased almost linearly with an increasing value of the MELD-Albumin score.

Actually, previous studies have demonstrated that the components of the MELD-Albumin score (total bilirubin, albumin, and $\mathrm{SCr}$ ) were associated with CA-AKI. Bilirubin is a physiologic antioxidant and free radical scavenger, ${ }^{19}$ which can protect renal function when the total bilirubin was mildly elevated within the normal range (laboratory normal range in our study was $0.1-1.0 \mathrm{mg} / \mathrm{dL}) .{ }^{20}$ However, $\mathrm{Wu}$ et al discovered that a higher level of total bilirubin was associated with an increased incidence of CA-AKI when the concentration of total bilirubin exceeded the normal range. ${ }^{20}$ It indicates that severe hyperbilirubinemia may induce the development of CA-AKI via reducing renal perfusion by decreasing the arterial pressure and intraglomerular pressure. ${ }^{21}$ In the formula of the MELDAlbumin score, the lower limit of total bilirubin was set at $1.0 \mathrm{mg} / \mathrm{dL}$, which is the upper limit of the normal range in our study; therefore, the value of the MELD-Albumin score elevated with increasing total bilirubin levels. Albumin, as a protein produced by the liver, is an important and recognized indicator of the synthetic function of the liver. ${ }^{22}$ The level of serum albumin reduces when the synthetic function of the liver decreases. Serum albumin has several functions including maintaining colloid osmotic pressure and increasing renal perfusion. ${ }^{23,24}$ Additionally, serum albumin also acts as an antioxidant by scavenging free radicals, ${ }^{25}$ which can reduce the risk of CA-AKI. Several studies have also detected a relationship between lower levels of serum albumin and CA-AKI. ${ }^{26-28}$ The MELDAlbumin score increased as the level of albumin decreased. $\mathrm{SCr}$ is used for the evaluation of renal function. Poor renal function has been shown to increase the risk of CA-AKI. ${ }^{29}$
In summary, the MELD-Albumin score combines these 3 parameters and can simultaneously evaluate liver and renal function. Our data showed that patients who developed CA-AKI have a higher level of aspartate aminotransferase (AST) and INR, and a lower level of serum albumin than patients who did not develop CA-AKI. Additionally, patients who developed CA-AKI have a higher incidence of CKD compared with those who did not develop CAAKI. Taken together, we consider that patients who developed CA-AKI may have a worse liver and renal function than those who did not develop CA-AKI. Besides, in the high MELD-Albumin score group (MELD-Albumin score >7.9), the level of AST, and INR was higher and the level of serum albumin was lower as compared to the low MELD-Albumin score group (MELD-Albumin score $\leq 7.9$ ). The incidence of CKD was also higher in the high MELDAlbumin score group (MELD-Albumin score $>7.9$ ) than in the low MELD-Albumin score group (MELD-Albumin score $\leq 7.9$ ) (Supplementary Table). It indicates that patients with a higher MELD-Albumin score are more likely to have a worse liver and renal function, which is associated with CA-AKI.

Apart from the mechanisms mentioned above, some factors that lead to liver injuries such as autoimmune disease, various drugs and toxins, and hepatitis $C$ virus, may trigger systemic inflammation and oxidative stress. ${ }^{30,31}$ This may ultimately result in CA-AKI because inflammation and oxidative stress are important pathophysiological mechanisms of CA-AKI. ${ }^{32}$ Additionally, the balance of vasoconstrictors and vasodilators is disrupted in the context of liver dysfunction, which causes splanchnic and systemic vasodilation and renal vasoconstriction. These conditions eventually lead to the impairment of renal function..$^{33}$ Therefore, the correction of liver function before the procedure has important clinical implications for improving patient outcomes.

As we mentioned above, the MELD score was originally developed to predict short-term poor prognosis of patients with cirrhosis who received a transjugular intrahepatic portosystemic shunt. ${ }^{16}$ Recently, a growing number of studies have demonstrated that the MELD-based scoring system could be used as a marker of cardiovascular risk in patients undergoing PCI, even in patients without end-stage liver disease. ${ }^{17,18,34}$ Individual components of the MELDAlbumin score (total bilirubin, albumin, and SCr) have been previously demonstrated to be associated with CAAKI. ${ }^{26,27,29,35}$ Furthermore, our study also discovered that the MELD-Albumin score was strongly associated with CA-AKI in patients undergoing elective PCI. Collectively, these data indicated that a cluster of risk factors included in the MELD-Albumin score might also provide potential predictive information on CA-AKI in the general population, including those without cirrhosis.

Several limitations exist in this study that need to be mentioned. First, the risk of selection bias cannot be ruled out due to the retrospective nature of our study. Second, we only calculated the MELD score and its modifications at a single time because the critical laboratory parameters included in the formula were measured only once. Therefore, random errors were inevitably introduced in the present study. Third, we did not include the etiology of liver dysfunction as a parameter in the analysis. The lack of relevant data limits the assessment of these variables. Fourth, as we mentioned above, autoimmune disease, various drugs and toxins, and hepatitis $\mathrm{C}$ virus may trigger systemic inflam- 
mation and oxidative stress. This may increase the risk of developing CA-AKI because inflammation and oxidative stress are important pathophysiological mechanisms of CA-AKI. However, we did not collect information about autoimmune disease, hepatitis $\mathrm{C}$ virus, and parameters reflecting inflammation and oxidative stress such as C-reactive protein, procalcitonin, and interleukin-6. Therefore, the mechanistic explanation for the association between the MELD-Albumin score and CA-AKI is speculative. Future studies are needed to further explore the specific mechanisms. Fifth, the long-term use of oral anticoagulants before admission may affect the measurement of INR, which could confound the results. However, we performed a sensitivity analysis by excluding patients who were taking warfarin from the main analysis and found that our main conclusions were not affected by these differences (data not shown). Finally, despite several confounding factors being adjusted for, the results of our study may have been subjected to residual confounding factors.

In conclusion, the MELD-Albumin score had the highest predictive value for predicting CA-AKI than other versions of the MELD score in patients undergoing elective PCI. There is a linear relationship between the MELDAlbumin score and the risk of CA-AKI. The addition of the MELD-Albumin score to the existing risk prediction model significantly improved the reclassification and discrimination ability for CA-AKI.

\section{Sources of Funding}

This work was supported by the National Natural Science Foundation of China General Program (Grant numbers: 81873495 and 82070375), the Heart Failure Center Research Fund of Fujian Provincial Hospital (supported by Fujian Provincial Department of Finance), the Natural Science Foundation of Fujian Province (2018J01242), the high-level hospital foster grants from Fujian Provincial Hospital, Fujian province, China (Grant number: 2020HSJJ05) and the Fujian provincial health technology project (Grant number: 2019-ZQN-10). The funding source had no role in this manuscript.

\section{Disclosures}

The authors declare that there are no conflicts of interest.

\section{IRB Information}

This study was approved by the ethics committee of Fujian Provincial Hospital (Ethical approval number: K2019-07-011).

\section{Data Availability}

The deidentified participant data will not be shared.

\section{References}

1. Weisbord SD, Palevsky PM, Kaufman JS, Wu H, Androsenko $\mathrm{M}$, Ferguson RE, et al. Contrast-associated acute kidney injury and serious adverse outcomes following angiography. J Am Coll Cardiol 2020; 75: 1311-1320.

2. Neyra JA, Shah S, Mooney R, Jacobsen G, Yee J, Novak JE. Contrast-induced acute kidney injury following coronary angiography: A cohort study of hospitalized patients with or without chronic kidney disease. Nephrol Dial Transplant 2013; 28: 1463 1471.

3. Aubry P, Brillet G, Catella L, Schmidt A, Bénard S. Outcomes, risk factors and health burden of contrast-induced acute kidney injury: An observational study of one million hospitalizations with image-guided cardiovascular procedures. BMC Nephrol 2016; 17: 167.

4. Alqahtani F, Balla S, AlHajji M, Chaudhary F, Albeiruti R, Kawsara A, et al. Temporal trends in the utilization and outcomes of percutaneous coronary interventions in patients with liver cirrhosis. Catheter Cardiovasc Interv 2020; 96: 802-810.

5. Lu DY, Steitieh D, Feldman DN, Cheung JW, Wong SC, Halazun $\mathrm{H}$, et al. Impact of cirrhosis on 90-day outcomes after percutane- ous coronary intervention (from a nationwide database). Am J Cardiol 2020; 125: 1295-1304.

6. Sujan R, Cruz-Lemini M, Altamirano J, Simonetto DA, Maiwall $\mathrm{R}$, Axley $\mathrm{P}$, et al. A validated score predicts acute kidney injury and survival in patients with alcoholic hepatitis. Liver Transpl 2018; 24: $1655-1664$.

7. Tariq R, Hadi Y, Chahal K, Reddy S, Salameh H, Singal AK Incidence, mortality and predictors of acute kidney injury in patients with cirrhosis: A systematic review and meta-analysis. $J$ Clin Transl Hepatol 2020; 8: 135-142.

8. Ding X, Xie H, Yang F, Wang L, Hou X. Risk factors of acute renal injury and in-hospital mortality in adult patients with postcardiotomy cardiogenic shock requiring veno-arterial extracorporeal membrane oxygenation: Utility of MELD-XI score. Perfusion, doi:10.1177/02676591211006619.

9. Cho E, Kim SC, Kim MG, Jo SK, Cho WY, Kim HK. The incidence and risk factors of acute kidney injury after hepatobiliary surgery: A prospective observational study. BMC Nephrol 2014; 15: 169

10. Bao B, Wang W, Wang Y, Chen Q. A prediction score model and survival analysis of acute kidney injury following orthotopic liver transplantation in adults. Ann Palliat Med 2021; 10: 6168 6179.

11. Mehta RL, Kellum JA, Shah SV, Molitoris BA, Ronco C, Warnock DG, et al. Acute Kidney Injury Network: Report of an initiative to improve outcomes in acute kidney injury. Crit Care 2007; 11: R31.

12. Fan H, Fan J, Chen S, Chen Y, Gao H, Shan L, et al. Prognostic significance of end-stage liver diseases, respiratory tract infection, and chronic kidney diseases in symptomatic acute hepatitis E. Front Cell Infect Microbiol 2021; 10: 593674.

13. Kim MS, Kato TS, Farr M, Wu C, Givens RC, Collado E, et al. Hepatic dysfunction in ambulatory patients with heart failure: Application of the MELD scoring system for outcome prediction. J Am Coll Cardiol 2013; 61: 2253-2261.

14. Szczurek W, Szyguła-Jurkiewicz B, Zakliczyński M, Król B, Gąsior M, Zembala M. Prognostic utility of the $\mathrm{N}$ terminal prohormone of brain natriuretic peptide and the modified Model for End Stage Liver Disease in patients with end stage heart failure. Pol Arch Intern Med 2018; 128: 235-243.

15. National Kidney Foundation. K/DOQI clinical practice guidelines for chronic kidney disease: Evaluation, classification, and stratification. Am J Kidney Dis 2002; 39(2 Suppl 1): S1-S266.

16. Malinchoc M, Kamath PS, Gordon FD, Peine CJ, Rank J, ter Borg PC. A model to predict poor survival in patients undergoing transjugular intrahepatic portosystemic shunts. Hepatology 2000; 31: 864-871.

17. Chen Y, Han M, Zheng YY, Zhu F, Aisan A, Maheshati T, et al. Model for end-stage liver disease score predicts the mortality of patients with coronary heart disease who underwent percutaneous coronary intervention. Cardiol Res Pract 2021; 2021: 6401092 .

18. Kırıs T, Avcı E, Çelik A. Combined value of left ventricular ejection fraction and the Model for End-Stage Liver Disease (MELD) score for predicting mortality in patients with acute coronary syndrome who were undergoing percutaneous coronary intervention. BMC Cardiovasc Disord 2018; 18: 44.

19. Neuzil J, Stocker R. Bilirubin attenuates radical-mediated damage to serum albumin. FEBS Lett 1993; 331: 281-284.

20. Wu YH, Wu CY, Cheng CY, Tsai SF. Severe hyperbilirubinemia is associated with higher risk of contrast-related acute kidney injury following contrast-enhanced computed tomography. PLoS One 2020; 15: $\mathrm{e} 0231264$.

21. Boon AC, Bulmer AC, Coombes JS, Fassett RG. Circulating bilirubin and defense against kidney disease and cardiovascular mortality: Mechanisms contributing to protection in clinical investigations. Am J Physiol Renal Physiol 2014; 307: F123-F136.

22. Arroyo V, García-Martinez R, Salvatella X. Human serum albumin, systemic inflammation, and cirrhosis. J Hepatol 2014; 61: 396-407.

23. Evans TW. Review article: Albumin as a drug--biological effects of albumin unrelated to oncotic pressure. Aliment Pharmacol Ther 2002; 16(Suppl 5): 6-11.

24. Lee EH, Kim WJ, Kim JY, Chin JH, Choi DK, Sim JY, et al. Effect of exogenous albumin on the incidence of postoperative acute kidney injury in patients undergoing off-pump coronary artery bypass surgery with a preoperative albumin level of less than $4.0 \mathrm{~g} / \mathrm{dl}$. Anesthesiology 2016; 124: 1001-1011.

25. Bourdon E, Blache D. The importance of proteins in defense against oxidation. Antioxid Redox Signal 2001; 3: 293-311. 
26. Murat SN, Kurtul A, Yarlioglues M. Impact of serum albumin levels on contrast-induced acute kidney injury in patients with acute coronary syndromes treated with percutaneous coronary intervention. Angiology 2015; 66: 732-737.

27. Liu L, Lun Z, Wang B, Lei L, Sun G, Liu J, et al. Predictive value of hypoalbuminemia for contrast-associated acute kidney injury: A systematic review and meta-analysis. Angiology 2021; 72: $616-624$

28. He HM, Zhang SC, He C, You ZB, Luo MQ, Lin MQ, et al. Association between neutrophil percentage-to-albumin ratio and contrast-associated acute kidney injury in patients without chronic kidney disease undergoing percutaneous coronary intervention. J Cardiol, doi:10.1016/j.jjcc.2021.09.004

29. Azzalini L, Ojeda S, Demir OM, Dens J, Tanabe M, La Manna A, et al. Recanalization of chronic total occlusions in patients with vs without chronic kidney disease: The impact of contrastinduced acute kidney injury. Can J Cardiol 2018; 34: 1275-1282.

30. DellaVolpe J, Al-Khafaji A. Acute kidney injury before and after liver transplant. J Intensive Care Med 2019; 34: 687-695.

31. Pfingstgraf IO, Taulescu M, Pop RM, Orăsan R, Vlase L, Uifalean
A, et al. Protective effects of Taraxacum officinale L. (Dandelion) root extract in experimental acute on chronic liver failure. Antioxidants (Basel) 2021; 10: 504.

32. Zhang F, Lu Z, Wang F. Advances in the pathogenesis and prevention of contrast-induced nephropathy. Life Sci 2020; 259: 118379.

33. Simonetto DA, Gines P, Kamath PS. Hepatorenal syndrome: Pathophysiology, diagnosis, and management. BMJ 2020; 370: $\mathrm{m} 2687$.

34. He SJ, Weng JX, Chen HJ, Li HQ, Guo WQ, Cao Q, et al. The prognostic value of MELD-XI in elderly patients with ST-segment elevation myocardial infarction: An observational study. $B M C$ Cardiovasc Disord 2021; 21: 53.

35. Vuruşkan E, Saraçoğlu E. Bilirubin levels are associated with contrast-induced nephropathy in peripheral artery disease. Angiology 2017; 68: $728-733$.

\section{Supplementary Files}

Please find supplementary file(s);

http://dx.doi.org/10.1253/circj.CJ-21-0816 